\title{
Surgical management of paraclinoid aneurysms via frontal lateral approach.
}

\author{
Jiantao Wang ${ }^{1,2}$, Zhisheng Kan ${ }^{1}$, Shuo Wang ${ }^{2 *}$ \\ ${ }^{1}$ Department of Neurosurgery, Beijing Anzhen Hospital, Capital Medical University, Beijing, PR China \\ ${ }^{2}$ Department of Neurosurgery, Beijing Tiantan Hospital, Capital Medical University, Beijing, PR China
}

\begin{abstract}
Objective: Towards exploring minimally invasive techniques in paraclinoid aneurysms clipping via a frontal lateral approach.

Methods: 118 patients by frontal lateral approach in the last $2.5 \mathrm{y}$ in Beijing Tiantan Hospital were analysed retrospectively. Clinical data and follow-up outcomes of the patients were reviewed. Digital Subtraction Angiography (DSA) was performed among all patients to categorize the paraclinoid aneurysms. The microsurgery through frontal lateral approach was performed in 118 patients. According to various subtypes of paraclinoid aneurysms, summarize and compare the surgical skills and outcome of the treatment.

Outcomes: Out of the 126 aneurysms, 116 got trimmed effectively, 5 was trapped. Numerous aneurysms in 3 incidents got cured in one craniotomy. Anterior clinoidectomy was removed in 110 cases. Aneurysm residues were found after 1 giant aneurysms clipping. One case lost his sight with preoperative visual light perception and in eight cases cerebral infarction occurred. Type I (dorsal projection) aneurysms constituted $47.5 \%$, Type II aneurysms (34.7\%) followed by Type III aneurysms constituted $17.8 \%$. All the others had a good recovery.

Conclusions: The paraclinoid aneurysm can be exposed and treated very well by microsurgery through frontal lateral approach which has the advantages such as minimal invasion, less effect on the patient's look and simple procedure. The lateral transfrontal approach is safe and effective in surgery for paraclinoid aneurysm. Among the 3 types, type I can reach better prognosis.
\end{abstract}

Keywords: Aneurysms, Digital subtraction angiography, Parasellar meningioma. Accepted on May 25, 2017

\section{Introduction}

Paraclinoid aneurysms are a challenging subset of intracranial aneurysms due to their surrounding anatomy. The section that distances the Internal Carotid Artery (ICA) wherefrom it comes out of the Cavernous Sinus (CS) and come into the subarachnoid area to the source of the subsequent collaborating vein is referred to as the carotid-ophthalmic section [1-5], the ophthalmic segment [6]. The C2 segment [7], and the paraclinoid segment [8-11]. These segment aneurysms are likely to be puzzling to cure through an exposed clinical approach subordinate to their closeness to the optical device and Anterior Clinoid Process (ACP). Ophthalmic section aneurysms make up around $5 \%$ of entire intracranial aneurysms and pose a clinical encounter due to the structural intricacy of the paraclinoid area, with closeness to the optic device, besides incomplete intracavernous leeway in certain sick persons $[6,12,13]$.

With the growth and modification of smallest clinical practices, however, the perils linked with straight clinical treatment have been diminished, and fruitful medication of paraclinoid aneurysms has turned out to be possible. In this reflective statement, we appraise and debate the classification and operational result of clinical cutting carried out by a lone surgeon in a sequence of 118 sick persons with 126 paraclinoid aneurysms through forward adjacent tactic.

\section{Methods}

\section{Clinical data}

The operative results in 118 sick persons with paraclinoid aneurysms were surveyed. Since July 2011 to October 2015,118 patients (89 women and 29 men, mean age $62 \mathrm{y}$, range 36 74 y) who had 126 ophthalmic artery aneurysms were admitted to Beijing Tiantan Hospital. Expositions for the 118 techniques indicated as under: accompanying (16.9\%), nuisance $(56.8 \%)$, visual symptoms (15.3\%), SAH $(7.6 \%)$, ischemic symptoms (3.4\%). In 9 sick persons with subarachnoid hemorrhages, the Hunt and Hess grade was I out of 5 patients, II out of 1 patient, and III out of 5 patients. All paraclinoid aneurysms were established consuming DSA. The entire sick persons endured preliminary operational calculated tomographic angiography using 3-measurable renovations for 
additional imagining of the association of the aneurysm to the skeletal construction. The measurement, form, and alignment of the aneurysms were evaluated applying DSA. The aneurysms were analysed into 3 clusters in accordance with DSA outcomes. Aneurysm measurement was analysed as minor $(<5 \mathrm{~mm})$, average $(5-10 \mathrm{~mm})$, big $(10-25 \mathrm{~mm})$, and huge $(>25 \mathrm{~mm})$.

\section{Frontal lateral approach}

The sick person is put in a flat place. The cranium is rotated by 30 degrees and stretched marginally at the neckline. The cervical carotid artery is organized and wrapped into the arena in case the nearby carotid uncovering is required to regulate. Incision (Figure 1a): It starts from edge of the hair line on forehead, 3-4 cm away from the center line, and goes downward along the internal arc of the hair line. It is about 8 $\mathrm{cm}$ in length, with its bottom $1.0 \mathrm{~cm}$ above the zygomatic arch. A 6-7 $\mathrm{cm}$ forehead arc incision can be marked along the edge of the hair line for those with low hair line; while the incision for those with high hair line or with scant hair can be marked along the forehead wrinkles. The skin, subcutaneous fascia and about $1 \mathrm{~cm}$ long temporalis should be cut open in sequence before pushing the periosteum. Then, the flap should be pulled to the superciliary arch side. While doing this, please note that the supra-orbital nerves and blood vessels should be protected. A bone window (Figure 1c) can be formed by drilling a hole on the temporal line (Figure 1b). An elliptic bone flap with an area of about $\left(3 \times 2.5 \mathrm{~cm}^{2}\right)$ should be shovelled down. The inferior border of the bone window should be in line with the anterior skull base. Front edge of the bone window is located 1.5-2.0 $\mathrm{cm}$ in front of the zygomatic process. The bone flap is near the skull base. Inner edge of the orbital bone window should be grinded smoothly, so as to improve the appearance of the forehead bottom. If the frontal sinus is open, it should be sealed with bone wax. The dura mater should be snipped along the edge of the orbit (taken as the basic arc) to open the Sylvian fissure cistern and slowly let out the cerebrospinal fluid (Figure 1d). After lifting the frontal pole and opening the carotid cistern and optic chiasma cistern, the same side optic nerve, optic chiasma and same side internal carotid and diaphragma sellae can be seen. We regularly begin close by regulation by uncovering the ICA in the neckline prepared prior to craniotomy in most ophthalmic section aneurysms. The Anterior Clinoid Process (ACP) is drilled intradurally for exposing proximal angle aneurysm to reveal the aneurysm fully (Figure 1e). The Dural rings are unlocked and the distanced hollow section of the ICA is uncovered if required. Multiple aneurysms can be attempted to clip via the same approach (Figure 1f). After clipping, Indocyanine Green (ICG) angiography was performed to reveal a leftover lot of the aneurysm post trimming or settlement of the unobstructed ICA uneasily ostensible beneath straight miniscule hallucination. DSA or CTA is performed regularly in $1 \mathrm{w}$.

\section{Classification of aneurysms}

The paraclinoid aneurysms stayed analysed into three clusters in accordance with a process stated by Barami et al. Type Ia and $\mathrm{Ib}$ aneurysms (Barami classification) were merged into a sole cluster (carotid ophthalmic). Type II aneurysms rising from the ventral ophthalmic section of the ICA were defined as ventral paraclinoid (ventral paraclinoid aneurysm). Type III aneurysms arising from the medial ophthalmic section of the ICA. The aneurysms associated to the larger hypophyseal vein were defined as larger hypophyseal.

\section{Results}

One hundred and twenty-one ophthalmic artery aneurysms (9 ruptured and 107 unruptured) were surgically managed by neck clipping (116 aneurysms) and wrapping [5] via frontal lateral approach at the Beijing Tiantan Hospital. When the aneurysms were unexposed due to the non-availability of information on the carotid cave, certain fragments of muscles were enfolded about the ICA at the source of the ophthalmic vein. Numerous aneurysms were discovered in $18(15.3 \%)$ sick persons. Sixtyfive aneurysms were 5 to $10 \mathrm{~mm}$, forty were $10-25 \mathrm{~mm}$, besides sixteen were more than $25 \mathrm{~mm}$ in size. Centered on the DSA discoveries, the sorts of such ophthalmic segment aneurysms were classified as $56(47.5 \%)$ aneurysms were Type I, while 41 (34.7\%) were Type II. 21 aneurysms (17.8\%) were Type III. Out of the patients with numerous aneurysms, eight had contralateral ophthalmic artery aneurysms and three (Type III) of them were managed by neck clipping via contralateral frontal lateral approach. Proximate CA regulation was acquired by uncovering the neck-related CA in 116 incidents $(98.3 \%)$. Postoperative CTA showed that no aneurysm which was cured using surgery warranted more therapy.

Sick persons' after operation position Glasgow Outcome Scale (GOS) besides pictorial and complete results were examined. The complete impediment rate of clinical therapy of aneurysms was truncated. Overall operative mortality was $0 \%, 88.9 \%$ good clinical outcome (GOS score of 5 and 4). Anatomical outcomes in for every subsection are abridged (Table 1). This follow-up time of 6 months to 28 months. Five sick persons with absence of previous pictorial indications had fresh pictorial shortfalls, post 6 months, they are recovered.

Table 1. Medical result in 118 paraclinoid aneurysms patients.

\begin{tabular}{llll}
\hline Time GOS & (Type) I & II & III \\
\hline $6-28$ months $(5+4)$ & $54(96.4 \%)$ & $35(85.3 \%)$ & $14(66.7 \%)$ \\
\hline$(3+2+1)$ & $2(3.6 \%)$ & $6(14.7 \%)$ & $7(33.3 \%)$ \\
\hline Total & 56 & 41 & 21 \\
\hline
\end{tabular}

The outcomes are articulated according to the GOS. A GOS score of 5 is an exceptional result, 4 is a decent result with adequate incapacity, 3 is a just result with simple incapacity, 2 is a meagre result (vegetative state), and 1 is lifeless. Commercially available software (SPSS version 11.5) was used for statistical analysis. A $\chi^{2}$ trial was applied for single 
change study to evaluate the affiliation among inconsistencies $\left(\chi^{2}=12.4011, \mathrm{P}<0.05\right)$.

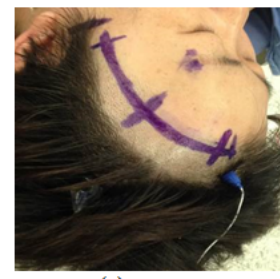

(a)

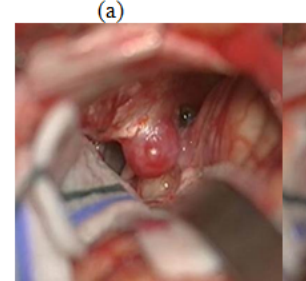

(d)

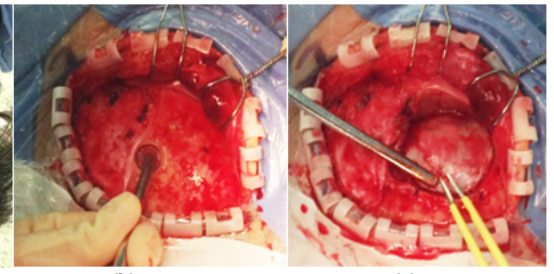

(b)

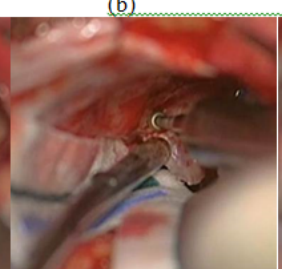

(e)

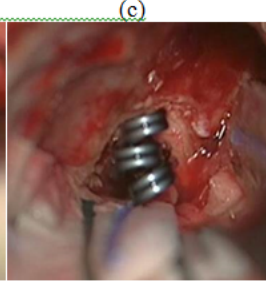

(f)

Figure 1. The ophthalmic aneurysm projecting medially was clipped successfully with side-angle straight clips by frontal lateral approach.
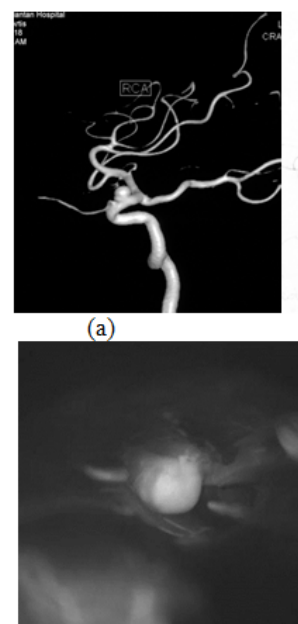

(d)
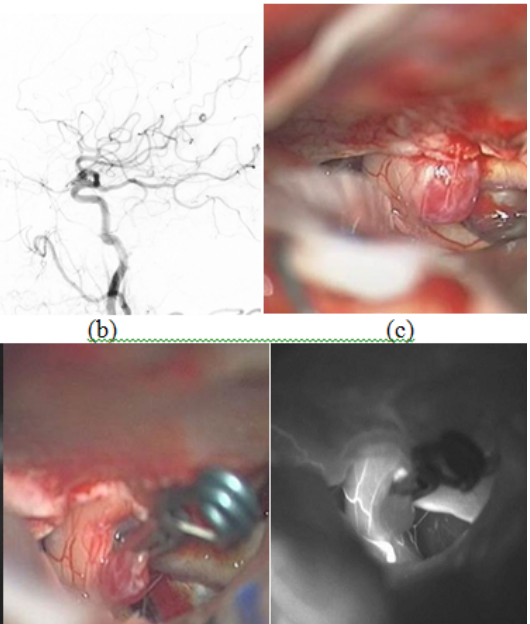

(e)

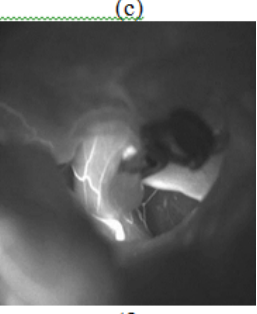

(f)

Figure 2. The paraclinoid aneurysm (Type I) was trimmed effectively with one direct clip.

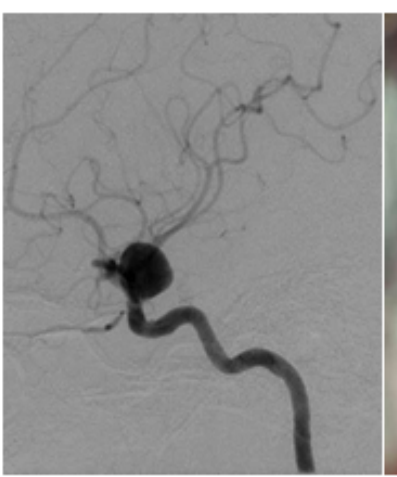

(a)

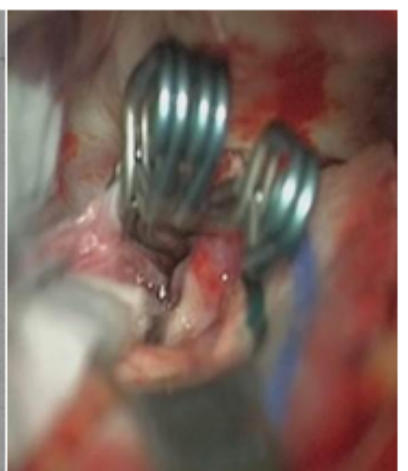

(b)

Figure 3. The paraclinoid aneurysm (ventral type) was clipped (Type II).

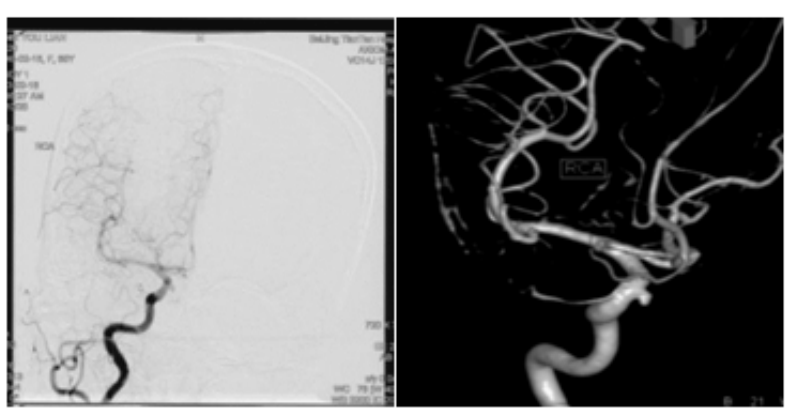

(a)

(b)

Figure 4. The paraclinoid aneurysm (ventral type) was clipped (Type III).

\section{Discussion}

In spite of progresses in prior to operation (CT angiography and MR simile) and intraoperative (angiography and intraoperative Doppler ultrasonography) neuroimaging besides technical enhancements (fluorescence compound microscope), operation still continues with the similar fundamental phases: proximate CA regulation, piercing the clinoid function, unwrapping the distance dural ring, unwrapping the falciform tendon and optic nerve channel, and carrying out particular trimming methods for every assembly of aneurysms $[5,6,9,10]$.

\section{Approach selection}

Brock and Dietz first reported treatment of intracranial aneurysm through small frontal bottom lateral approach [14]. After that, there are many scholars successively adopting this approach to incise various tumors including anterior communicating artery aneurysm, pituitary tumor, craniopharyngioma, parasellar meningioma and olfactory groove meningioma. Frontal lateral approach is an operative approach between supraorbital keyhole approach and pterional approach. It not only has some advantages of keyhole approach of small incision, little bleeding and quick recovery; but also has the advantages of normal pterional approach of broad view and convenient operation for microsurgery. The pterional approach and frontal lateral approach adopt the surgical approach of smooth skull base by getting deep into skull from the natural anatomic space between anterolateral surface of the frontal lobe and the skull base which is separately $1 \mathrm{~cm}$ ahead and behind the frontal bone zygomatic process. There is no distinctive difference in their effect on exposing structures such as anterior circulation aneurysm, parental artery perforator vessels and nerves, etc. [15]. Compared with pterion approach, the frontal lateral approach reduces invalid exposure of brain tissues, and avoids dura artery injury. Meanwhile, there is no need to grind away the sphenoid ridge and temporal squama below the projection line of the lateral fissure, which saves time for opening and closing the skull, avoids unnecessary bleeding, and at the same time, with small injury to temporalis, avoids the side powerless occlusion due to postoperative muscle atrophy, and collapsed appearance of the temple [16]. Frontal lateral approach should be with bone flap as low as 
possible and front edge of forehead side bone window near the skull base; part of the inner plate and diploe can be tilted and grinded to augment the exposure area toward inside of the skull. Our treatment of aneurysm on ophthalmic artery through frontal lateral approach has achieved a satisfactory effect, with the clipping effect not worse than the pterional approach and no notable differences in operative complications which are similar to those described in foreign literatures $[15,17]$. Contrasted with the pterion tactic, the frontal lateral tactic has the following benefits: 1. Relatively small incision; 2 . Small incision of temporalis or no need of temporalis incision; 3. Small bone window, without need to grind sphenoid ridge, retaining the sphenoid ridge and reducing the opened area on both sides of the forehead-temple so as to minimize the bone loss caused by craniotomy, and get good bone flap reposition to better meet with the dissection and physiological status; 4 . Retaining of sphenoid ridge and temporal squama below the projection line of the lateral fissure, reducing the exposure of orbit and dura artery, and middle meningeal artery groove, and avoiding unnecessary bleeding; 5. Reduced time for surgery. It also has disadvantages: it's more appropriate for sick persons with clipping aneurysm with Hunt-Hess grade lower than level III, but due to limited exposure view, it's difficult to treat with operative accident like massive hemorrhage during operation; for sick persons with Hunt-Hess grade between levels IV V , in a critical condition with high intracranial pressure needing to enlarge bone window to reduce pressure, the approach can be properly enlarged. IV and V level patients often have intracranial hematoma at the same time. Sometimes they even show early-stage cerebral hernia, then, the hematoma should be eliminated immediately, and the bone flap should be cut to reduce pressure. It's not suitable to do outside forehead miniinvasive clipping to the aneurysm [18]. Since the sphenoid ridge is not grinded away, and the Sylvian fissure is only exposed with its front $1 / 3$, this approach is not suitable for middle cerebral artery aneurysm. However, the exposure area has been enough for aneurysm on ophthalmic artery. The frontal lateral approach isn't purely in pursuit of small bone window, but it retains necessary exposure for operated area and reduces "unnecessary exposure" for brain tissues. With small space for operation, once aneurysm rupture occurs during the operation, it's difficult to implement vascular occlusion, thus, the surgeon should have excellent skill in micromanipulation [19]. A neck-related skin slit is normally done prior to craniotomy to protect the cervical ICA except the aneurysm is placed much over head the distance ring. If the aneurysm was ruptured in the craniotomy or separation procedure, temporary blocked carotid artery can reduce tension of aneurysm, soften and collapse the aneurysm adequately, which in favor of the separation and clipping the aneurysm. Lately, proximate regulation has typically been accomplished by a straight clinical tactic and unlikely to be acquired, particularly when the aneurysm is jutting imperiously (Type I) and positioned much on the frontal clinoid function. In this group, the cervical skin incision is made for temporary occlusion except two (Type I) cases (1.7\%).

\section{ACP resection}

Prior to the progress of smallest clinical cranial base tactics, the ACP clogged the neurosurgeon's vision and the direction of outbreak applied to cure the aneurysm. It is one of the obstacles in clipping these aneurysms. We need more space for the blade into the aneurysm clip, between the aneurysm neck and the ACP. In our estimation, an enhanced precision fastener location with fewer ordeal to the pictorial method and its vascular delivery is delivered if this movement is executed totally, rather than attempting to place a clip to a constricted predicament with incomplete imagining. Prior to operation 3D CT angiography with deduction processes assist to idea the degree of piercing and clip positioning. Delicate microsurgical processes like piercing the ACP, dividing of the optic nerve cover, and utilization of the optic nerve may be needed for optimum treatment of complex or lower position aneurysm [20]. Elimination of the ACP allows imagining of around 6 $\mathrm{mm}$ more of the proximate ICA devoid of entering the echoing sinus [21]. In the current research, the anterior clinoid process was detached intradurally by applying a rapid diamond borer in 113 cases $(95.7 \%)$; the only exclusions were two sick persons with Type I cuts and three patients with Type III lesions which were clipped via contralateral approach. There were two methods for ACP resection (intradurally and extradurally) [22-24]. There are many conflicts on if clinoidectomy was to be performed extradurally or intradurally. We trust that the benefits of carrying out the frontal clinoidectomy post dural unwrapping is the visualization one can have on the prediction of the aneurysm and close by neuro-vascular assemblies. The process of ACP cutting out can be carried out under straight hallucination to prevent unnecessary excessive bone reduction. Occasionally, the whole clinoid is not required to be eliminated to see the proximate neckline. At our institution, we remain strong proponents of an intradural anterior clinoidectomy for ophthalmic segment aneurysms. It provides improved exposure of ophthalmic segment aneurysms and protection of oculomotor nerve. There was no oculomotor nerve damage occurred in this group. The falciform ligament is cut, enabling deployment of the cranial fiber bundle. The relief of the falciform ligament and deploying the cranial fiber bundle can assist in defining the aneurysm besides the proximate part of the neckline of the aneurysm. Precise care must be given to development of air cells and a link with the sphenoid sinus after boring the ACP and cranial support. When the ACP and optic strut are pneumatized, we support padding with a plump implant, and a minor pericranial implant stitched in place. This method may avert CSF rhinorrhea. For contralateral ophthalmic section aneurysms, elimination of the frontal clinoid function or removing the roof of the cranial fiber did not occur [25]. Two aneurysms endured cutting via a contralateral tactic at the time of cutting of the ipsilateral aneurysm. We have located countless worth in getting preoperative competent tomographic angiograms with three measurement renovations of the optic $f$ to authenticate the association of the aneurysm to the skeletal structure. With three-dimensional reconstructions, we can find the relationship between ACP and aneurysm to guide surgery operation. For 5 
patients in this group, after review CTA image before surgery, it's estimated the aneurysm is difficult to be clipped. After negotiation with their relatives, we adopted wrapping of the aneurysm.

\section{Microsurgical clipping}

Cutting of paraclinoid aneurysms can be very perplexing. Assuming their measurement and the bordering with bone and vascular fiber assemblies, we applied a wide range of clips, many of them oblique or provided with window, but also other types in accordance with aneurysm kind.

Type I: Superior aneurysm projection. From a clinical standpoint, commandingly protrusive (Figure 2) are calmest to cure, having hardly any affiliation with commanding hypophysial vessels or other tactically vital vessels. Often, only the cranial fiber and the OphA vein should be assigned. These cuts are clinically calmer to uncover than other C6 aneurysms [26]. Here, a slanting clip is hosted over the ICA from the adjacent side with the vanes of the clasp directing towards the middle and lowly, or a right-angled clasp is used along the ICA from the proximate side in parallel with the vanes of the clasp directing towards the distance. If aneurysms were wide built and immobile and hence were tough to eliminate. Deploying the carotid vein with circumferential separation of the distanced Dural round trailed by proximate and distanced provisional cutting loosened the section of the carotid sufficient to enable acceptable location of the clasp.

Type II (ventral paraclinoid aneurysms): In incidents, wherein the aneurysm proposes in inferior way (Figure 3), the aneurysmal neckline was seen ventral to the ICA in the subdural area, and the proximate part of the aneurysmal neckline and the cupola were positioned in the hollow sinus. These aneurysms are micro clinically tougher to uncover. To enable mild medial withdrawal of type II aneurysms, the distanced dural ring along the surface of the cranial channel was cut, and the hollow section was deployed crosswise for proximate regulation. The rectilinear fenestrated clasp can be used in a matching manner with the $\mathrm{CA}$ in the fenestration. Classically, a right-angled fenestrated clip was applied. Moreover, clasp obstruction of carotid cave aneurysms (Group II) needs additional broad piercing of the basal bone assembly, thus hypothetically enhancing the clinical peril. Besides, when the dimension of the aneurysm enhances, there could be a better likelihood of unfinished clasp obstruction due to the connection among the clasp vane and the basal bone assembly. For aneurysm, unable to be uncovered totally, they should not be cut compulsorily, so covering should be espoused. Applying to cover aneurysm, 5 patients in this cluster are not able to uncover the aneurysm totally.

Type III: The aneurysm was found medial to the ICA (Figure 4). For these Group aneurysms, additional widespread cutting out the frontal clinoid function and cranial support is required to uncover the aneurysm for clasp positioning. Prior to the operation, it used to be normally cumbersome to decide if a type III aneurysm was jutting above or below the diaphragma sella. To view the supradiaphragmatic different, the cranial fiber cover was slit and the cranial fiber was lightly withdrawn in the middle. The supradiaphragmatic different aneurysm was usually trimmed with a right angled fenestrated clasp operated from an adjacent course. Since the infradiaphragmatic different was normally under the diaphragm, it was not imagined in spite of adjacent movement of the ophthalmic section of the ICA. In such a scenario, the distanced dural ring was cut marginally round the carotid vein and protracted in the middle diagonally the diaphragma sella, deserting a manacle of the dura fastened to the border of the aneurysm. A right angle bent fenestrated clasp (kept surrounding the ICA) successfully eliminated the aneurysm. Bipolar tongs can be made use of to shrink the aneurysm to enable trimming. Besides, selecting a suitably measured with shape of clips is more cumbersome to get total destruction without compromising the clinoidal (C-3) part of the CA. Since the proximal portion of the aneurysm neck was not completely occluded, the side-angel straight clip, oblique-angled ring clip or a blend of fenestrated and straight clasp were used to cut the aneurysm. When opening clasps are applied, it is greatest to overvalue the quantum of left over carotid vein lumen needed to uphold the expansion.

For huge cuts, such a tandem preparation may not bear adequate concluding potency to ruin the pouch. Provisional catching of the aneurysm and puncture of the pouch can be carried out, nonetheless a securer substitute could be to keep two or three lengthy, big-orifice, direct clasps right-angled from the bloc of the tandem vanes. Provisional obstruction permits the pouch to become softer. Post this occurrence, bipolar tongs could be applied to level these "load baggage," to conclude, curbing the neckline and enabling the initially chosen clasp spans sufficient for the work. As and when likely, straight elimination of the aneurysm is the utmost preferred cure tactic. Especially for paraclinoid aneurysms which were characterized in superiorly projecting, large or giant, wide neck $[27,28]$.

\section{Visual outcome}

For patients with preoperative change in visual sense or with large area aneurysm, after temporary blocking of the internal carotid, the aneurysm should be punctured to pump the thrombus or cut open to take out the thrombus. After the volume of aneurysm being reduced, the clipping can be done on the aneurysm, which is useful for postoperative recovery of visual sense. A pictorial theme fault was observed in 5 sick persons and development was observed in 3 sick persons. Of the 13 patients presenting with diminished visual acuity, 10 had enhanced pictorial insight. Largely, pictorial standing enhancement was noticed in 13 of the 18 sick persons $(72.2 \%)$.

\section{Opposite side of body cuts}

It is common for ophthalmic vein aneurysms to be two-sided, and attention may be provided to the likelihood of trying to protect both cuts through the similar tactic [29]. Frontal lateral approach can be implemented through rotating the bed for operation, which is easier for incising the aneurysm under the optic nerve, especially the inclined offside aneurysm. The 
frontal lateral approach can provide an enough outside space under forehead. Regarding exposing tissues in sellar region, it can fully expose visual nerves on both sides, optic chiasma cistern, lateral surface of same side internal carotid cistern and medial surface of opposite side internal carotid cistern, medial wall of cavernous sinus. In our opinion, there are few attributes of the contraleteral aneurysm that enable the same tactic. The lesion must clearly be unsplit, since proximate regulation may be tough. The aneurysm must initiate on the higher or middle of the founder carotid vein (Types I and III), simplifying the reach beneath the contralateral cranial fiber. The aneurysm must be trivial that minimum cutting up is essential; in the proposed research, the corresponding author might obviously envisage the neckline of the conflicting aneurysm in 3 sick persons and hence trimmed through a contralateral tactic. Among them, one case had a huge frontal interactive vein aneurysm, combined through contralateral ophthalmic vein aneurysm (Type III). The other 2 cases had a posterior communicating artery aneurysm associated with contralateral ophthalmic artery. The 3 sick persons had eye-related section aneurysm and other aneurysms trimmed with one surgical opening into the skull.

\section{Indications of trap}

Contrasting utmost aneurysms, such paraclinoid aneurysms ascend at a place unconnected to exact major divisions. In this zone, aneurysms can be intradural, extradural, or both; there is absence of dependable X-ray study to turn this fortitude viable. In this group, there were 5 aneurysms cannot be clipped which were wrapped, because the 5 aneurysms may be partially located in the hollow sinus and the aneurysm neckline cannot fully revealed.

\section{Conclusions}

The complex anatomy of the paraclinoid ICA makes the surgical management of aneurysms arising from this segment difficult. The key features of successful surgical treatment of these lesions include establishing control of the proximal artery, adequate exposure of the aneurysm neck, and successful obliteration of the aneurysm with minimal manipulation of the optic nerve. Accurate preoperative assessment of the origin of these lesions is critical for determining the surgical approach. The advantages of the frontal lateral tactic comprise less functioning time, less skin shock, less confinement in hospital, lesser expenses and improved visual and practical outcomes. It can meet the operating requirements of the paraclinoid aneurysms. Medical wounding is a real therapy with good clinical results besides satisfactory impediment magnitudes in experts' hands. Medical results differed meaningfully among Groups I and others. These differences reach statistical significance. Type I can reach better Prognosis. Anterior clinoidectomy was the necessary process for most of ophthalmic artery aneurysms to sufficient uncover the aneurysm neckline and the parent vein.

\section{References}

1. Almeida GM, Shibata MK, Bianco E. Carotid-ophthalmic aneurysms. Surg Neurol 1976; 5: 41-45.

2. Dolenc VV. A combined epi- and subdural direct approach to carotid-ophthalmic artery aneurysms. J Neurosurg 1985; 62: 667-672.

3. Dolenc VV. A combined transorbital-transclinoid and transsylvian approach to carotid-ophthalmic aneurysms without retraction of the brain. Acta Neurochir Suppl (Wien) 1999; 72: 89-97.

4. Kumon Y, Sakaki S, Kohno K, Ohta S, Ohue S, Oka Y. Asymptomatic, unruptured carotid-ophthalmic artery aneurysms:Angiographical differentiation of each type, operative results, and indications. Surg Neurol 1997; 48: 465-472.

5. Yasargil MG, Gasser JC, Hodosh RM, Rankin TV. Carotidophthalmic aneurysms: Direct microsurgical approach. Surg Neurol 1977; 8: 155-165.

6. Day AL. Aneurysms of the ophthalmic segment. A clinical and anatomical analysis. J Neurosurg 1990; 72: 677-691.

7. Krayenbu H, Yasargil MG. Rontegenanatomie und topographie der hirngefasse, in Huber P (eds): Zerebrale Angiographie fur Klinik und Praxis. Stuttgart Georg Thieme 1979; 238-246.

8. Arnautovic KI, Al-Mefty O, Angtuaco E. A combined microsurgical skull-base and endovascular approach to giant and large paraclinoid aneurysms. Surg Neurol 1998; 50: 504-520.

9. Batjer HH, Kopitnik TA, Giller CA, Samson DS. Surgery for paraclinoidal carotid artery aneurysms. J Neurosurg 1994; 80: 650-658.

10. De Jesus O, Sekhar LN, Riedel CJ. Clinoid and paraclinoid aneurysms: Surgical anatomy, operative techniques, and outcome. Surg Neurol 1999; 51: 477-488.

11. Kattner KA, Bailes J, Fukushima T. Direct surgical management of large bulbous and giant aneurysms involving the paraclinoid segment of the internal carotid artery: Report of 29 cases. Surg Neurol 1998; 49: 471-480.

12. Batjer HH, Kopitnik TA, Giller CA, Samson DS. Surgery for paraclinoidal carotid artery aneurysms. J Neurosurg 1994; 80: 650-658.

13. Ferguson GG, Drake CG. Carotid-ophthalmic aneurysms: visual abnormalities in 32 patients and the results of treatment. Surg Neurol 1981; 16: 1-8.

14. Brock M, Dietz H. The small frontolateral approach for the microsurgical treatment of intracranial aneurysms. Neurochirurgia 1978; 21: 185-191.

15. Figueiredo EG, Deshmukh P, Nakaji P. The minipterional craniotomy:technical description and anatomic assessment. Neurosurgery 2007; 61: 256-264.

16. Czirja SGT. Surgical experience with frontolateral keyhole craniotomy through a superciliary skin incision. Neurosurgery 2001; 48: 145-150.

17. Paladino J, Mrak G, Mikli P. The key hole concept in aneurysm surgery? a comparative study: keyhole versus 
standard craniotomy. Minim Invasive Neurosurg 2005; 48: 251-258.

18. Lan Q, Gong Z, Kang D. Microsurgical experience with keyhole operations 0n intracranial aneurysm. Surg Neurol 2006; 66: 2 .

19. Yeremeyeva E, Salma A, Chow A. Microscopic and endoscopic antefior communicating artery complex anatomy as seen through keyhole approaches. J Clin Neurosci 2012; 19: 1422.

20. Park SK, Shin YS, Lim YC. Preoperative predictive value of the necessity for anterior clinoidectomy in posterior communicating artery aneurysm clipping. Neurosurgery 2009; 65: 281-285.

21. Nutik SL. Removal of the anterior clinoid process for exposure of the proximal intracranial carotid artery. J Neurosurg 1988; 69: 529-534.

22. Lehmberg J, Krieg SM, Meyer B. Anterior clinoidectomy. Acta Neurochir (Wien) 2014; 156: 415-419.

23. Lai LT, Morgan MK. Outcomes for unruptured ophthalmic segment aneurysm surgery. J Clin Neurosci 2013; 20: 1127-1133.

24. Spektor S, Dotan S, Mizrahi CJ. Safety of drilling for clinoidectomy and optic canal unroofing in anterior skull base surgery. Acta Neurochir (Wien) 2013; 6: 1017-1024.

25. Hopf NJ, Stadie A, Reisch R. Surgical management of bilateral middle cerebral artery aneurysms via a unilateral supraorbital key-hole craniotomy. Minim Invasive Neurosurg 2009; 3: 126-131.
26. Hoh B, Carter B, Budzik R. Results after surgical and endovascular treatment of paraclinoid aneurysms by a combined neurovascular team. Neurosurgery 2001; 48: 78-89.

27. Barami K, Hemandez VS, Diaz FG, Guthikonda M. Paraclinoid carotid aneurysms:surgical management,complications, and outcome based on a new classification scheme. Skull Base 2003; 13: 31-41.

28. Shefif C, Grnber A, Doffer C. Ruptured carotid artery aneurysms of the ophthalmic(C6) segment: clinical and angiographic long term follow up of a multidisciplinary management strategy. J Neurol Neurosurg Psychiatry 2009; 80: 1261-1267.

29. Kakizawa Y, Tanaka Y, Orz Y, Iwashita T, Hongo K, Kobayashi S. Parameters of contralateral approach to ophthalmic segment aneurysms of the internal carotid artery. Neurosurgery 2000; 47: 1130-1137.

\section{*Correspondence to}

Shuo Wang

Department of Neurosurgery

Beijing Tiantan Hospital

Capital Medical University

PR China 